'Facultad de Medicina, Pontificia Universidad Católica de Chile. Santiago, Chile.

2Departamento de Anatomía Patológica, Facultad de Medicina, Pontificia Universidad Católica de Chile. Santiago, Chile.

${ }^{3}$ Departamento de Dermatología, Facultad de Medicina, Pontificia Universidad Católica de Chile. Santiago, Chile. ${ }^{4}$ Departamento de Cirugía Oncológica, Facultad de Medicina, Pontificia Universidad Católica de Chile. Santiago, Chile.

${ }^{5}$ Departamento de Oncología, Facultad de Medicina, Pontificia Universidad Católica de Chile. Santiago, Chile.

6Unidad de Melanoma y Cáncer de Piel UC, Facultad de Medicina, Pontificia Universidad Católica de Chile. Santiago, Chile. ${ }^{a}$ Alumno

Recibido el 30 de julio de 2015, aceptado el 13 de abril de 2016.

Correspondencia a: Dr. Pablo Uribe González Depto. de Dermatología Unidad de Melanoma y Cáncer de Piel UC. Facultad de Medicina Pontificia Universidad Católica de Chile. Av. Vicuña Mackenna 4686, primer piso. Macul, Santiago. Tel: $56-2-23548492$ Fax: $56-2-25529974$ puribeg@med.puc.cl

\section{Entendiendo las terapias actuales en melanoma metastásico}

\author{
ROCÍO RODRÍGUEZ ${ }^{1, a}$, ANGELA PARRA ${ }^{1, a}$, SERGIO GONZÁLEZ $Z^{2,5}$, \\ MONTSERRAT MOLGÓ ${ }^{3,6}$, NICOLÁS DROPPELMANN ${ }^{4,6}$, \\ FRANCISCO ACEVEDO ${ }^{5,6}$, JOSÉ PEÑA ${ }^{5}$, PABLO URIBE ${ }^{3,6}$

\section{Understanding current therapies in metastatic melanoma}

Cutaneous melanoma is a highly aggressive tumor developing from melanocytes, its incidence is increasing, and prognosis in advanced stages is daunting. New therapies have been approved during the recent years with unprecedented results, including inhibitors of MAPK/ERK pathway and immune checkpoint blockade (anti-cytotoxic T lymphocyte antigen-4 (CTLA-4) as ipilimumab, anti-programmed cell death protein 1 (PD-L1) as pembrolizumab and anti-programmed cell death protein 1 ligand (PD-L1), among many others). The aim of this paper is to review currently available metastatic melanoma therapies focusing mainly on new therapies that have demonstrated effectiveness, after several decades of little progress in the treatment of this disease.

(Rev Med Chile 2016; 144: 1448-1458)

Key words: CTLA-4 Antigen; Melanoma; Molecular Targeted Therapy; Pembrolizumab; Programmed Cell Death 1 Receptor.

\begin{tabular}{|ll|}
\hline CTLA-4: & Antígeno 4 del linfocito T citotóxico \\
DTIC: & Dimetil Triazeno Imidazol Carboxamida \\
EGFR: & Receptor del factor de crecimiento epidérmico \\
ERK: & Quinasa regulada por señal extracelular \\
FDA: & Administración de drogas y alimentos \\
LT: & Linfocito T \\
MAPK: & Proteína quinasa activada por mitógenos \\
PD1: & Proteína de muerte celular programada \\
PDL-1: & Ligando proteína de muerte celular programada \\
RR: & Riesgo relativo \\
SLP: & Sobrevida libre de progresión \\
SG: & $\quad$ Sobrevida global \\
TCR: & Receptor de linfocito T \\
\hline
\end{tabular}

1 1 melanoma cutáneo es un tumor muy agresivo desarrollado a partir de melanocitos. Representa aproximadamente el $75 \%$ de las muertes relacionadas al cáncer de piel $^{1} \mathrm{y}$ su incidencia está en aumento, con 73.870 casos nuevos estimados para 2015 en Estados Unidos 2. En Chile, para los años 2003-2007, se estimó una tasa de incidencia de 2,7 (hombres) y 2,2 (mujeres) por 100.000 habitantes; la tasa de mortalidad se estimó para el año 2012 en 0,7 por $100.000^{3,4}$.

El pronóstico de melanoma depende de varios factores, entre ellos espesor tumoral de Breslow, ulceración, mitosis, compromiso de linfonodos regionales y metástasis a distancia, variables consideradas en la clasificación $\mathrm{TNM}^{5}$. En etapas iniciales tiene una sobrevida a 5 años de $98 \%$, en cambio en pacientes con melanoma metastásico la sobrevida a 5 años es solo $16 \%{ }^{6}$. El pilar del manejo del melanoma cutáneo localizado es la cirugía, que cura a la mayoría de los pacientes, pero un porcen- 
Tabla 1. Terapias actuales en el tratamiento de melanoma metastásico

\begin{tabular}{|c|c|}
\hline Grupo Farmacológico & Comentarios por grupo farmacológico \\
\hline \multicolumn{2}{|l|}{ 1. Quimioterapia } \\
\hline 2. Inhibidores Farmacológicos de vías moleculares & Medicamentos de uso oral \\
\hline Inhibidores de BRAF (BRAFi): & Requieren mutaciones BRAF \\
\hline - Vemurafenib (Zelboraf®, Roche, FDA 2012) & Respuesta rápida antitumoral \\
\hline - Dabrafenib (Tafinlar®, GSK, FDA 2013) & Resistencia \\
\hline \multicolumn{2}{|l|}{ Inhibidores de MEK (MEKi): } \\
\hline \multicolumn{2}{|l|}{ - Trametinib (Mekinist®, GSK, FDA 2013) } \\
\hline \multicolumn{2}{|l|}{ - Cobimetinib (Cotellic®, Roche, FDA 2015) } \\
\hline \multicolumn{2}{|l|}{ Combinación BRAFi+ MEKi } \\
\hline \multicolumn{2}{|l|}{ - Dabrafenib + Trametinib (FDA 2014) } \\
\hline \multicolumn{2}{|l|}{ - Vemurafenib + Cobimetinib (FDA 2015) } \\
\hline 3. Inhibidores de puntos de control inmune & Anticuerpos Monoclonales \\
\hline Anti CTLA-4: & Uso endovenosos $c / 2-4$ sem \\
\hline - Ipilimumab (Yervoy®, BMS, FDA 2011) & No requieren mutaciones BRAF \\
\hline Anti PD-1: & Respuesta más lenta (excepto combinación) \\
\hline - Nivolumab (Opdivo®, BMS, FDA 2014) & Prolongada \\
\hline \multicolumn{2}{|l|}{ - Pembrolizumab (Keytruda®, Merck, FDA 2014) } \\
\hline \multicolumn{2}{|l|}{ Combinación anti CTL4 + anti PD1 } \\
\hline - Ipilimumab + Nivolumab (FDA 2015) & \\
\hline
\end{tabular}

taje no despreciable desarrollará eventualmente enfermedad irresecable o metástasis ${ }^{7}$. Tradicionalmente, en etapas metastásicas, el melanoma es raramente curable. Las nuevas aproximaciones terapéuticas han demostrado beneficios clínicos, principalmente inhibidores de vías moleculares e inhibidores de puntos de control inmune, que hoy están desplazando a la quimioterapia citotóxica como principal herramienta terapéutica para el melanoma metastásico.

A continuación revisaremos las terapias de uso actual en melanoma metastásico (Tabla 1), centrándonos en los nuevos tratamientos que han resultado eficaces luego de varias décadas de escaso progreso en el tratamiento de esta enfermedad.

\section{Quimioterapia}

El primer hito en el tratamiento del melanoma avanzado ocurrió en 1975, cuando la dacarbazina (DTIC) fue aprobada por FDA. Agente citostático alquilante que se asocia a tasas de respuesta mo- destas (12\%), y no se ha comparado con placebo, por lo que no hubiese sido aprobada por la FDA bajo los estándares actuales ${ }^{8}$. DTIC -y su prodroga temozolomida- han sido los agentes quimioterapéuticos estándares en el tratamiento del melanoma, por lo que son el punto de comparación para muchas de las terapias recién desarrolladas ${ }^{9}$.

Aunque otros agentes han mostrado actividad en melanoma metastásico (ej. cisplatino, taxanos), ninguno logró reemplazar a DTIC ${ }^{10,11}$. Asimismo, el uso de combinaciones de quimioterapia citotóxica, si bien se asocia a mayor respuesta, es más tóxica y no impacta en la sobrevida ${ }^{12,13}$. Esto se debe a la alta tasa de quimiorresistencia del melanoma por alteración en transportadores que provocan eflujo de las drogas, desintoxicación aumentada, mutación de topoisomerasa y particularmente por mayor resistencia a la apoptosis ${ }^{14-17}$.

Actualmente en el manejo inicial de los pacientes con melanoma metastásico, se prefiere el uso de otros agentes ${ }^{18}$ como mencionaremos a continuación. 
Inhibidores farmacológicos de vías moleculares: inhibidores de vía MAPK ERK

Las neoplasias se originan por la acumulación de alteraciones en la función de genes críticos, que alteran la regulación de la proliferación, diferenciación y muerte celular entregando ventaja de crecimiento en comparación con otras poblaciones celulares $^{19,20}$. La vía de señalización MAPK RAS/ RAF/MEK/ERK es esencial en procesos celulares fundamentales, como proliferación, diferenciación, migración y apoptosis de los melanocitos ${ }^{21}$. Esta vía se encuentra activada por variados mecanismos en melanoma, siendo lo más frecuente la mutación BRAF. El bloqueo de esta vía de señalización mediante la unión selectiva a alguna de las proteínas quinasa de la cascada MAPK/ERK lleva a la inhibición de su fosforilación y de su translocación al núcleo de fosfo-Erk, reduciéndose las transcripción de genes importantes en aumento de sobrevida celular, angiogénesis y reducción de la apoptosis, por lo que se ha convertido en un objetivo terapéutico primordial en melanoma metastásico (Figura 1).

\section{a) Inhibidores de BRAF (BRAFi)}

Mutaciones activantes de $B R A F$ están presentes en aproximadamente un 50-60\% de los melanomas avanzados, especialmente ubicados en áreas no fotoexpuestas crónicamente ${ }^{22,23}$. Más del $70 \%$ de las mutaciones en BRAF son V600E (reemplaza valina por ácido glutámico en el aminoácido 600) y entre $10-30 \%$ son V600K (valina por lisina $)^{24}$. Estas mutaciones determinan una

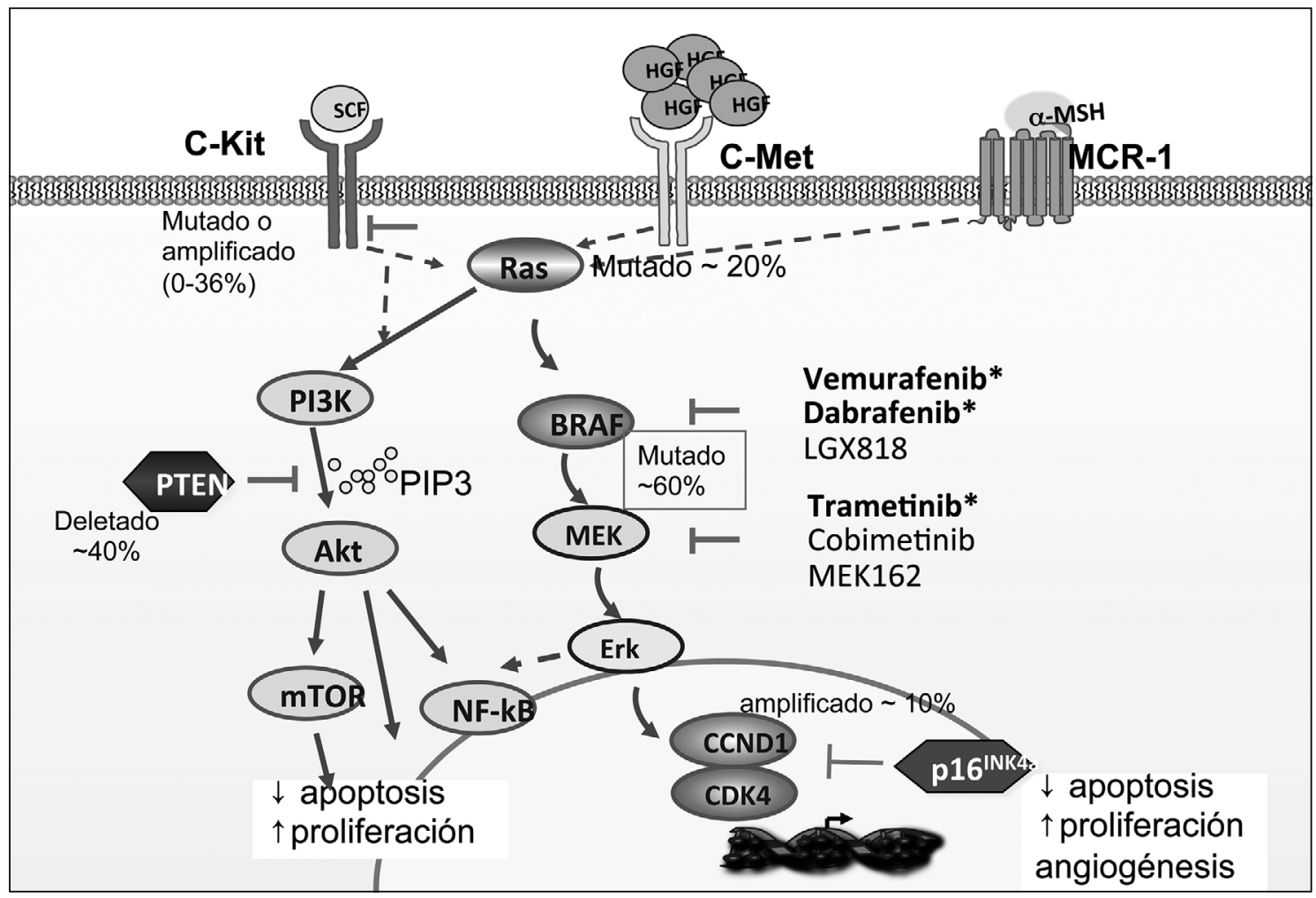

Figura 1. Varios oncogenes y vías moleculares son objetivo de las nuevas terapias en melanoma. La vía con mayor frecuencia alterada es MAPK RAS/RAF/MEK/ERK que controla procesos celulares como crecimiento, diferenciación y apoptosis; 50-60\% de los melanomas cutáneos tienen mutaciones activantes de BRAF y 15-20\% mutaciones en RAS, este último también activa la vía PI3K/PTEN/AKT que controla la proliferación celular. Esta segunda vía en 40-60\% de los melanomas interrumpe la expresión anormal de PTEN, cuya expresión aberrante también resulta en aumento de los niveles de AKT activado. La tercera vía de oncogenes clásica en melanoma es c-KIT que activa las vías MAPK y PI3K/AKT descritas anteriormente. En la figura se muestran también drogas que bloquean un determinado componente de las vías, por ejemplo BRAF o MAPK. 
activación permanente de la señalización de la vía MAPK, siendo tumorogénicas in vitro e in vivo y un blanco terapéutico en melanoma. Inicialmente se utilizaron BRAFi no selectivos, como sorafenib, demostrándose bajas tasas de respuesta ${ }^{25,26}$. Afortunadamente, la capacidad para detectar mutaciones de $B R A F$ en material histopatológico y la disponibilidad de inhibidores específicos de $B R A F$ mutado han cambiado el manejo de pacientes con melanoma ${ }^{27}$.

Vemurafenib (Zelboraf ${ }^{\circledR}$ ) y dabrafenib (Tafin$\left.\operatorname{lar}^{\circledR}\right)$ son inhibidores orales específicos con alta actividad contra BRAF V600E y V600K, y actividad variable ante otras mutaciones de BRAF (Tabla 1). Actúan uniéndose al sitio de unión del ATP de B-Raf mutado, inhibiendo la forma activa de la quinasa. Estos medicamentos mostraron excelentes resultados en estudios fase III en pacientes con melanoma irresecable o metastásico ${ }^{28-31}$. Se estima que la tasa de respuesta completa a ambos agentes es del 50-60\%, con una sobrevida libre de progresión (SLP) de 7 meses, llevando la sobrevida global (SG) a 14-16 meses. Estos resultados favorables también se han visto en pacientes con peor pronóstico (etapa M1c o altos niveles de LDH) ${ }^{28}$, incluso en aquellos con metástasis cerebrales ${ }^{30}$. Lamentablemente, todos desarrollarán resistencia al tratamiento ${ }^{32}$ debido a mecanismos que incluyen alteraciones en proteínas que conforman las vías de las MAPK como mutaciones de NRAS, amplificación de $B R A F$, expresión de variantes truncas de la proteína, aumento de niveles de $\mathrm{CRAS}^{33}$, activación de receptores tirosina quinasa y activación o mutación de $\mathrm{MEK}^{34,35}$, justificándose el uso de inhibidores de MEK (ver más adelante). También está descrita la activación de otras vías de señalización, como PI3K-AKT ${ }^{36}$.

Los BRAFi se asocian a algunos efectos adversos, lo que se explica en parte por activación paradójica de la vía MAPK en células normales (ej. queratinocito), resultando en queratoderma plantar, queratosis pilaris, queratosis verrucosas y carcinoma escamoso tipo queratoacantoma (19$26 \%)^{28}$. Estas lesiones aparecen pocas semanas después de iniciado el tratamiento y no determinan su interrupción ${ }^{37,38}$. También se observan modificaciones en lesiones pigmentadas; cambios en color, tamaño, erupción de múltiples nevos y aparición de nuevos melanomas primarios ${ }^{39,40}$. Otro efecto adverso relevante es fotosensibilidad a UVA, que sólo ocurre con vemurafenib ${ }^{41,42}$.

\section{b) Inhibidores de MEK (MEKi)}

MEK es un componente de la vía MAPK que se encuentra "río abajo" de la vía de $B R A F$, disponiéndose actualmente de 2 inhibidores selectivos de MEK1/2: trametinib y cobimetinib ${ }^{28}$. Trametinib (Mekinist ${ }^{\circledR}$ ) fue aprobado por FDA el 2013 como tratamiento de primera línea en pacientes con melanoma metastásico con mutaciones BRAF V600E y V600K. Según el estudio en fase III la SLP es de 4,8 meses para trametinib comparado con 1,5 meses con quimioterapia, mejorando la SG en $14 \%$ a 6 meses ${ }^{43}$. Cobimetinib (Cotellic $\left.{ }^{\circledR}\right)$, fue aprobado recientemente para ser utilizado en combinación con vemurafenib (ver más adelante $)^{44,45}$.

En un $87 \%$ de los pacientes que utilizan MEKi se observan efectos adversos cutáneos, similares a los inducidos por inhibidores de EGFR, especialmente erupción acneiforme, xerosis, paroniquia $y$ fisuras acrales ${ }^{46-48}$. La frecuencia de carcinoma escamoso cutáneo no aumenta ${ }^{43}$. Otros efectos adversos incluyen diarrea, edema periférico, fatiga, náuseas y más raramente disfunción ventricular, problemas visuales y enfermedad pulmonar intersticial ${ }^{9}$.

\section{c) Terapia combinada \\ Dabrafenib (BRAFi) + trametinib (MEKi)}

En un esfuerzo por retrasar el desarrollo de resistencia al tratamiento (que ocurre 6 a 7 meses de iniciada la terapia $)^{31}$ y minimizar la toxicidad asociada a la inhibición de BRAF, se estudió el uso combinado de dabrafenib y trametinib, aprobándose por la FDA en 2014 como terapia inicial en pacientes cuyo melanoma contiene la mutación BRAF V600E o V600K. Esta aprobación extendida se basa en estudios fase I/II donde mejoró la SLP, de 5,8 meses para dabrafenib como agente único versus 9,8 meses en terapia combinada.

\section{Vemurafenib (BRAFi) + cobimetinib (MEKi)}

La terapia combinada aumenta la SLP a 11,3 meses comparada con 7,3 meses con vemurafenib, y SG a 12 meses de $72 \%$ y $65 \%$, respectivamente ${ }^{49,50}$. Esta combinación fue aprobada por FDA el 2015 para pacientes con melanoma irresecable o metastásico con mutación en BRAF V600E o V600K.

El uso de terapia combinada disminuye los efectos adversos cutáneos en comparación con la monoterapia, debido a la reducción de la activa- 
ción paradójica de la vía MAPK. Esta activación ocurre en células que no presentan la mutación de BRAF, por ejemplo, queratinocitos normotípicos, queratinocitos con displasia, nevos melanocíticos, etc. Se produce por la unión de BRAFi a la proteína BRAF nativa, permitiendo la dimerización con otra proteína (CRAF) y siendo este complejo el que activa paradójicamente la vía MAPK. La adición de un MEKi (ej: trametinib) a un BRAFi (ej: dabrafenib), bloquea la activacion paradójica, reduciendo el desarrollo de lesiones cutáneas hiperproliferativas, tales como carcinoma de células escamosas ( $1 \%$ en terapia combinada versus $18 \%$ en monoterapia con dabrafenib), queratosis verrucosas ( $2 \%$ versus $23 \%$ ), queratodermia ( $4 \%$ versus $25 \%$ ) y teóricamente también disminuye la posibilidad de segundas neoplasias secundarias a la activación paradójica de MAPK. También existe una reducción notable de la frecuencia de erupciones acneiformes ${ }^{46,47,51}$, pero un incremento en la frecuencia y severidad de fiebre secundaria al tratamiento.

\section{Inhibidores de puntos de control inmune}

El melanoma posee una alta capacidad inmunogénica y la respuesta inmune del paciente puede influir en su historia natural. Esto se refleja en los fenómenos de regresión de las lesiones primarias, la respuesta a citoquinas como IL2 y la mayor incidencia de melanoma en pacientes inmunosuprimidos. Para estimular ésta respuesta inmune se han desarrollado vacunas, pero sus resultados son variables, no logrando aún demostrar beneficio en sobrevida por lo que son consideradas aún en experimentación. En Chile se desarrolló TAPCells, que logró inducir una respuesta antitumoral efectiva en estudios fase I/II; faltan estudios fase III para definir el impacto y la utilidad clínica de esta terapia ${ }^{52,53}$.

El problema en melanoma es que a pesar de la generación de una respuesta inmune, no se logra una actividad anti tumoral adecuada, debido a mecanismos que se describen a continuación.

La respuesta inmune celular se desarrolla luego que un determinado antígeno, por ejemplo un antígeno asociado al tumor (AAT), es presentado por una célula presentadora de antígenos (CPA) a un linfocito T (LT). Esta sinapsis inmunológica es compleja y en ella existen varias señales activa- doras/represoras complementarias (Figura 2A). Inicialmente, la CPA (ej. célula de Langerhans) procesa el antígeno y lo expone en su superficie mediante el complejo mayor de histocompatibilidad II (MHC-II), siendo reconocido por el LT, a través de su receptor TCR (señal 1). La segunda señal puede ser coactivadora o coinhibitoria. La señal coactivadora ocurre cuando B7 (receptor en CPA, también llamado CD80/CD86) se une a CD28 (LT); en esas condiciones el LT se activa, prolifera, madura y circula en la periferia, donde eventualmente se encontrará con el antígeno que le fue presentado, y ejercerá su efecto. Por el contrario, como medida para proteger las células sanas, minimizando la activación no específica de LT y el desarrollo de autoinmunidad, existe coinhibición a través de la unión de B7 a CTLA-4 (antígeno 4 del linfocito $\mathrm{T}$ citotóxico, expresado en células $\mathrm{T}$ activadas), impidiendo la interacción CD28-B7 e inhibibiendo la activación de LT. Además, CTL-4 produce activación los LT regulatorios, involucrados en la tolerancia inmunológica. En melanoma hay un estado anérgico con una mayor expresión de CTLA-4 (señal co-inhibitoria), como forma de evadir la activación de células $\mathrm{T}$ antitumorales (Figura 2A).

Por otro lado, se ha descubierto otro mecanismo inhibidor de la respuesta inmune antitumoral, a nivel periférico, en la interacción del LT efector y la célula tumoral: las células cancerosas expresan constitutivamente ligandos, por ejemplo PD-L1, que al unirse a su receptor ( $\mathrm{PD}-1$, proteína de muerte celular programada) disminuye la activación del LT efector (Figura 2B $)^{54}$.

Actualmente, hay estrategias inmunoterapéuticas que bloquean CTLA-4 y PD-1 ("checkpoints" del sistema inmune), mediante anticuerpos monoclonales, restableciendo y potenciando mecanismos antitumorales del sistema inmune a expensas de un aumento en la incidencia de fenómenos autoinmunes.

\section{a) Anti CTLA-4: ipilimumab y tramelimumab}

Ipilimumab (Yervoy ${ }^{\circledR}$ ) es un anticuerpo monoclonal humanizado con alta afinidad a CTLA-4 (Figura 2A). Aprobado en 2011 por FDA, luego de un estudio fase III que demostró, RR de 10,9\%, control de enfermedad (pacientes con respuesta parcial, completa o enfermedad estable) de $28,5 \%{ }^{55}$ y SG de $23,5 \%$ a los 24 meses en pacientes con tratamiento previo. Por primera vez un 
estudio clínico fue capaz de demostrar beneficio en sobrevida global en pacientes con melanoma metástasico. Este beneficio fue independiente de la etapa, LDH basal o uso previo de IL2.

Se han buscado marcadores de respuesta que permitan identificar los pacientes que se beneficiarán del tratamiento con ipilimumab; sin embargo, ninguno se encuentra validado y en uso clínico ${ }^{56,57}$.

Un aspecto importante del tratamiento con Ipilimumab es que puede producir patrones de respuesta que no se observan al usar agentes citotóxicos y que han sido definidos en los Criterios de Respuesta relacionados a la Inmunidad ("Immunerelated response criteria”). Los patrones son: a) respuesta en lesiones medibles a la semana 12 de tratamiento, sin aparición de nuevas lesiones; b) enfermedad estable (en algunos pacientes seguida de lenta disminución del tamaño tumoral); c) aumento inicial de tamaño tumoral, seguido de respuesta; d) Reducción en carga tumoral total, ya sea durante o después de la aparición de nuevas lesiones, después de la semana $12^{58}$.

Un $10-15 \%$ de los pacientes en tratamiento con Ipilimumab sufren efectos adversos grado 3 o 4. En su mayoría autoinmunes y se manifiestan como colitis, hepatitis, hipofisitis y manifestaciones dermatológicas como prurito y rash (maculopapulares y liquenoides $)^{59,60}$.

\section{b) Anticuerpos monoclonales Anti PD-1: \\ Nivolumab, Pembrolizumab}

Nivolumab (Opdivo $\left.{ }^{\circledR}\right)$. Aprobado el 2014 por FDA para pacientes previamente tratados con Ipilimumab o BRAFi (Figura 2B), y recientemente como primera línea en pacientes con melanoma avanzado o metastásico sin mutación de BRAF. Un estudio de fase III realizado en pacientes con melanoma sin tratamiento previo y sin mutación $B R A F$ evidenció RR de $40 \%$ y OS a 1 año de $72,9 \%$ con nivolumab, lo cual se comparó contra DTIC, que logró $13,9 \%$ y $42,1 \%$, respectivamente ${ }^{61}$. La continuación de este estudio mostró SG con nivolumab de 57,7\%, SLP media de 5,4 meses versus SG de $26,7 \%$ y SLP de 2,2 meses con DTIC. Respuesta completa de $11 \%$ de los pacientes con nivolumab y $1 \%$ con $\mathrm{DTIC}^{62}$. Por otro lado, nivolumab fue comparado con quimioterapia en pacientes con melanoma avanzado que progresaron luego de tratamiento con ipilimumab o ipilimumab y un inhibidor de BRAF en casos de mutación de este último; se vio mejor RR (31,7\% vs 10,6\%) y menos efectos adversos grado 3 o 4 (5 vs 9\%) con el uso de nivolumab ${ }^{63}$.

Pembrolizumab (Keytruda ${ }^{\circledR}$ ). Aprobado en 2014 por FDA para pacientes con fracaso al tratamiento con ipilimumab o un BRAFi, en casos de $B R A F$ mutado y recién aprobado como tratamiento de primera línea en pacientes con melanoma avanzado no tratado, independiente de mutación $B R A F$; logra RR de 38 a 52\%, la mayoría de estas respuestas son duraderas y no varían si hay exposición previa a ipilimumab ${ }^{64}$.

Pembrolizumab fue comparado con Ipilimumab, en primera línea de tratamiento, en un estudio fase III; logró mejor RR (33\% vs $11,9 \%$ ), SLP a 6 meses ( $47 \%$ versus $26,5 \%$ ) y OS $(74 \%-68 \%$ versus $58 \%$ en 12 meses). Con ambos tratamientos las respuestas fueron duraderas (alrededor de 90\% con una mediana de seguimiento de 7,9 meses), pero pembrolizumab tuvo menos efectos adversos grado 3 a $5^{65}$. Estos resultados favorables ocurren independiente del estado funcional, nivel de $\mathrm{LDH}$, mutación $B R A F$, etapa TNM y número o tipos de tratamientos previos ${ }^{66}$.

En general, el tratamiento con antiPD-1 es bien tolerado, con efectos adversos leves como fatiga, náuseas, diarrea y prurito; y algunos de naturaleza autoinmune como vitíligo $(10 \%)$ y rash no especifico (15\%). En menos del $2 \%$ de los casos se presentan efectos adversos grado 3 y 4 como trastornos tiroideos, colitis, hipofisitis, hepatitis y neumonitis ${ }^{61,63}$.

\section{c) Anticuerpos anti PD-L1.}

Están en investigación, aún no aprobados por FDA al momento de la revisión. Los más importantes, BMS-936559 y MPDL3280A, han mostrado RR de $17 \%$ y $26 \%$ en estudios preliminares ${ }^{67-69}$.

\section{d) Combinación de tratamientos. Ipilimumab (Anti CTLA-4) + nivolumab (anti PD-1)}

Aprobado por FDA el 2015 para pacientes con melanoma no resecable o metastásico y sin mutaciones de BRAF. Estudios de fase II/III muestran que esta combinación tiene mayor actividad antitumoral en comparación a la monoterapia con Ipilimumab, con RR de $61 \%$ versus $11 \%{ }^{70}$ y SLP significativamente mayor, independiente del estado mutacional de BRAF; 11,5 meses con el tratamiento combinado, 2,9 meses con ipilimumab y 6,9 meses con nivolumab ${ }^{71}$. Es destacable que hubo 11,5 a $22 \%$ de respuesta completa en el grupo con 


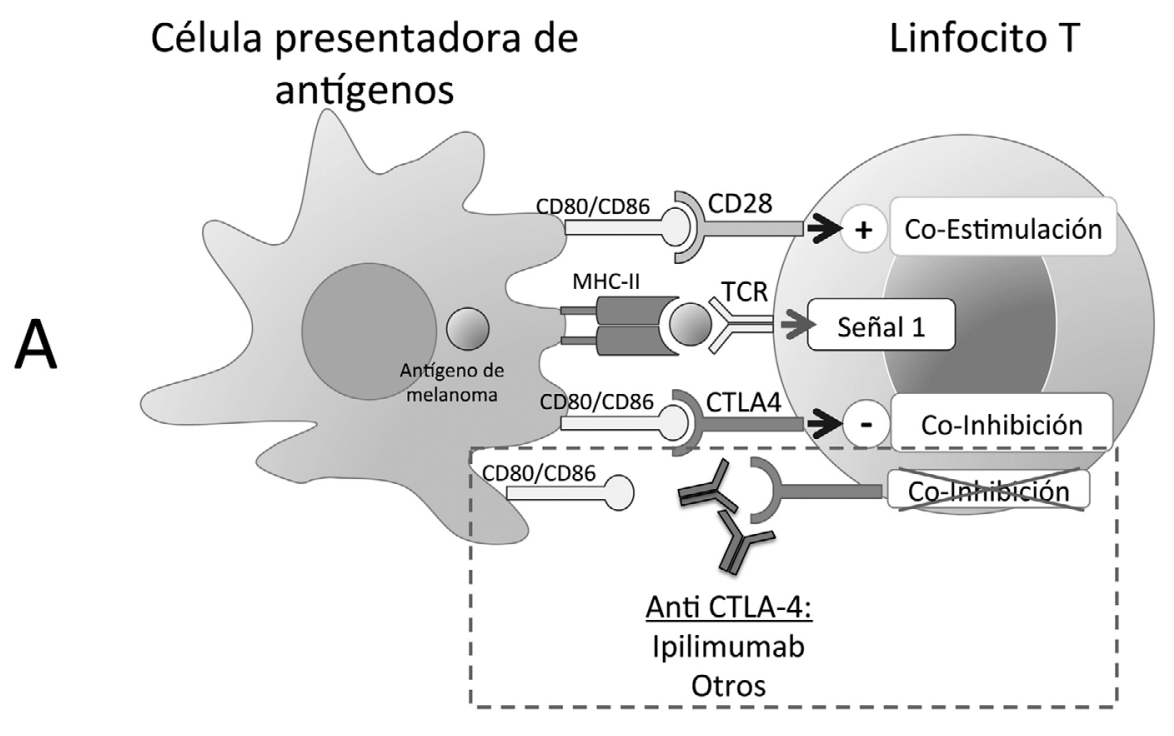

Melanoma $\quad$ Linfocito T citotóxico

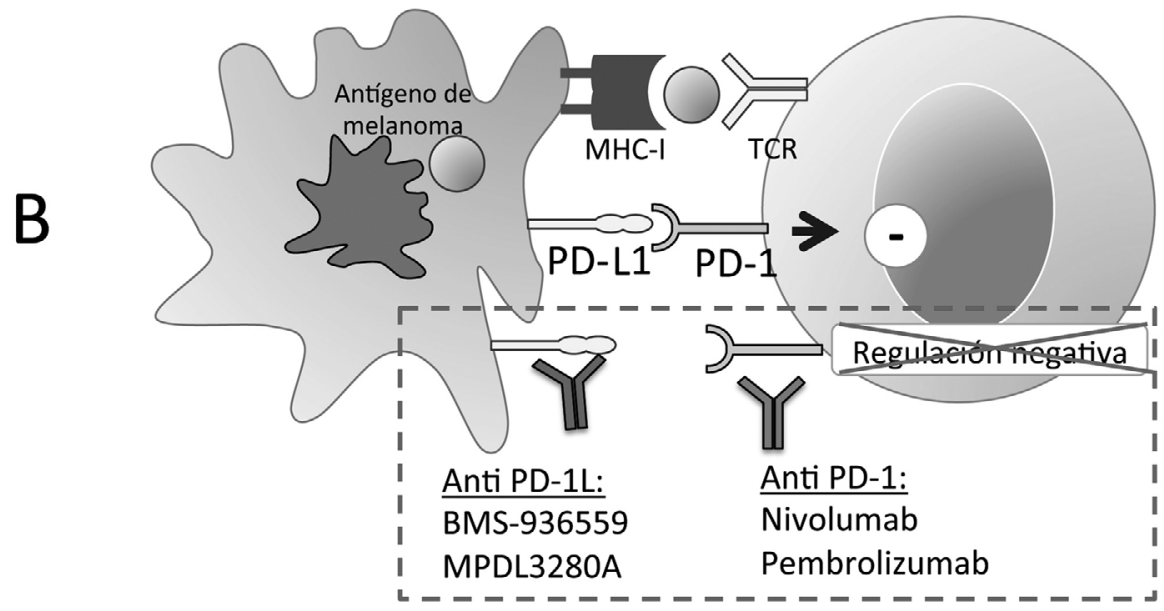

Figura 2. (A) Para la activación del LT la CPA mediante MHC-II, presenta el antígeno al linfocito T que lo reconoce a través de TCR (señal 1), y el receptor de la célula presentadora CD80/86 se une al receptor CD28 del linfocito T (señal 2). Luego de este proceso se expresa CTLA-4 en la superficie del linfocito T que se une con mayor afinidad a CD80/86 y produce una inhibición de la actividad del linfocito $T$, resultando en menor actividad antitumoral en melanoma. La inmunoterapia actualmente dispone antiCTLA-4 se une al receptor respectivo en el linfocito T e impide su unión a CD80/86 restaurando la activación de esta célula y permitiendo su acción antitumoral con resultados alentadores. (B) Células de melanoma expresan constitutivamente PD-1 que al unirse a su receptor PD-L1 presente en linfocitos T efectores, regulan de forma negativa la acción de esta célula, por lo que anticuerpos desarrollados contra estos blancos, hoy disponibles, permiten mejorar la respuesta autoinmune antitumoral. MHCII: complejo mayor de histocompatibilidad II; LT: linfocito T, CPA: Célula presentadora de antígenos; TCR: Receptor de linfocito T; CTLA-4: antígeno 4 del linfocito T citotóxico; PD1: proteína de muerte celular programada; PDL1: ligando de proteína de muerte celular programada. 
terapia combinada, $8,9 \%$ con nivolumab y sólo $2,2 \%$ con ipilimumab. En relación a la sobrevida se ha objetivado con la terapia combinada valores de $85 \%$ a 1 año y $79 \%$ a 2 años $^{72}$. La toxicidad es manejable, pero se observaron mayores efectos adversos grado 3 y 4 en el tratamiento combinado (54\% vs $24 \%$ ) destacando colitis y elevación de alanina aminotransferasa, entre otros ${ }^{70,71,73}$.

\section{Estrategias de tratamiento en pacientes con melanoma metastásico}

Están evolucionando constantemente y actualmente el esquema de tratamiento apunta a ser personalizado, dependiendo principalmente de 3 características ${ }^{74}$ :

1. Paciente, sus comorbilidades y estado funcional.

2. Las metástasis, su cantidad, ubicación, resecabilidad y síntomatología.

3. Mutaciones genéticas conocidas ("estado mutacional”): BRAF mutado u otras mutaciones.

En pacientes con un número limitado de metástasis, estables y potencialmente resecables, se debe considerar la metastasectomía, la que ha mostrado tasas de sobrevida a 5 años de hasta $40 \%{ }^{75}$.

En caso de lesiones irresecables o mayor número de metástasis, se recomienda iniciar terapias sistémicas y para decidir cual utilizar se debe determinar el estado funcional del paciente y estudiar presencia de mutaciones $B R A F$.

Si no hay mutaciones conocidas o el paciente tiene mutaciones asociadas a buen estado funcional, se debe considerar utilizar monoterapia con antiPD-1 o combinación anti-PD1 e ipilimumab. Si el estudio mutacional es positivo pero hay pobre capacidad funcional y/o alta carga metastásica, o si la enfermedad progresa a pesar de la inmunoterapia, se recomienda iniciar terapia molecular combinada: BRAFi+ MEKi ${ }^{74}$.

\section{Conclusiones}

El conocimiento de la biología molecular del melanoma y el desarrollo de inhibidores de vías moleculares e inmunoterapia han revolucionado el tratamiento del melanoma metastásico.
En comparación con la terapia citotóxica, estos fármacos logran mejorar las tasas de respuesta, sobrevida libre de progresión y sobrevida global, con perfiles de toxicidad aceptables, pero aún con un alto costo. En el futuro, algunos biomarcadores predicirán la respuesta a estos tratamientos, mejorando la relación costo-beneficio de estos medicamentos. Actualmente, se están realizando múltiples ensayos clínicos usando combinación de estos tratamientos, siendo algunas altamente efectivas y se estima que en algunos años un subgrupo de pacientes con melanoma metastásico se comportará como portadores de una enfermedad crónica.

\section{Referencias}

1. Olszanski AJ. Current and future roles of targeted therapy and immunotherapy in advanced melanoma. J Manag Care Pharm 2014; 20 (4): 346-56.

2. Siegel RL, Miller KD, Jemal A. Cancer statistics, 2015. CA Cancer J Clin 2015; 65 (1): 5-29.

3. Minsal, Unidad de Vigilancia de Enfermedades No transmisibles y Estudios, Departamento de Epidemiología, División de Planificación Sanitaria Subsecretaría de Salud Pública. Primer Informe de Registros Poblacionales de Cáncer de Chile, quinquenio 2003-2007. 2012. Disponible en: epi.minsal.cl [Consultado el 11 de Mayo de 2015].

4. GLOBOCAN International Agency for Research on Cancer. World Health Organization. Estimated cancer Incidence, mortality and prevalence Worldwide in 2012, 2012. Disponible en: http://globocan.iarc.fr. [Consultado 11 de Mayo de 2015].

5. Balch CM, Gershenwald JE, Soong SJ, Thompson JF, Atkins MB, Byrd DR, et al. Final version of 2009 AJCC melanoma staging and classification. J Clin Oncol 2009; 27 (36): 6199-206.

6. AmericanCancerSociety. Cancer Facts \& Figures 2014. (Society AC, ed). Atlanta. 2014.

7. Romano E, Scordo M, Dusza SW, Coit DG, Chapman $\mathrm{PB}$. Site and timing of first relapse in stage III melanoma patients: implications for follow-up guidelines. J Clin Oncol 2010; 28 (18): 3042-7.

8. Middleton MR, Grob JJ, Aaronson N, Fierlbeck G, Tilgen W, Seiter S, et al. Randomized phase III study of temozolomide versus dacarbazine in the treatment of patients with advanced metastatic malignant melanoma. J Clin Oncol 2000; 18 (1): 158-66.

9. Shah DJ, Dronca RS. Latest advances in chemotherapeutic, targeted, and immune approaches in the treatment 
of metastatic melanoma. Mayo Clin Proc 2014; 89 (4): 504-19.

10. Anderson CM, Buzaid AC, Legha SS. Systemic treatments for advanced cutaneous melanoma. Oncology (Williston Park) 1995; 9 (11): 1149-58; discussion 63-4, 67-8.

11. Mays SR, Nelson BR. Current therapy of cutaneous melanoma. Cutis 1999; 63 (5): 293-8.

12. Chapman PB, Einhorn LH, Meyers ML, Saxman S, Destro AN, Panageas KS, et al. Phase III multicenter randomized trial of the Dartmouth regimen versus dacarbazine in patients with metastatic melanoma. J Clin Oncol 1999; 17 (9): 2745-51.

13. Eigentler TK, Caroli UM, Radny P, Garbe C. Palliative therapy of disseminated malignant melanoma: a systematic review of 41 randomised clinical trials. Lancet Oncol 2003; 4 (12): 748-59.

14. Rockmann H, Schadendorf D. Drug resistance in human melanoma: mechanisms and therapeutic opportunities. Onkologie 2003; 26 (6): 581-7.

15. Grossman D, Altieri DC. Drug resistance in melanoma: mechanisms, apoptosis, and new potential therapeutic targets. Cancer Metastasis Rev 2001; 20 (1-2): 3-11.

16. La Porta CA. Drug resistance in melanoma: new perspectives. Curr Med Chem 2007; 14 (4): 387-91.

17. Stahl JM, Cheung M, Sharma A, Trivedi NR, Shanmugam S, Robertson GP. Loss of PTEN promotes tumor development in malignant melanoma. Cancer Res 2003; 63 (11): 2881-90.

18. Sosman J. Cytotoxic chemotherapy for metastatic melanoma. En: UpToDate. 2014.

19. Davies H, Bignell GR, Cox C, Stephens P, Edkins S, Clegg S, et al. Mutations of the BRAF gene in human cancer. Nature 2002; 417 (6892): 949-54.

20. Millington GW. Mutations of the BRAF gene in human cancer, by Davies, et al. (Nature 2002; 417: 949-54). Clin Exp Dermatol 2013; 38 (2): 222-3.

21. Dhillon AS, Hagan S, Rath O, Kolch W. MAP kinase signalling pathways in cancer. Oncogene 2007; 26 (22): 3279-90.

22. Long GV, Menzies AM, Nagrial AM, Haydu LE, Hamilton AL, Mann GJ, et al. Prognostic and clinicopathologic associations of oncogenic BRAF in metastatic melanoma. J Clin Oncol 2011; 29 (10): 1239-46.

23. Wellbrock C, Hurlstone A. BRAF as therapeutic target in melanoma. Biochem Pharmacol 2010; 80 (5): 561-7.

24. Klein O, Clements A, Menzies AM, O’Toole S, Kefford RF, Long GV. BRAF inhibitor activity in V600R metastatic melanoma. Eur J Cancer 2013; 49 (5): 1073-9.

25. Eisen T, Ahmad T, Flaherty KT, Gore M, Kaye S, Marais $\mathrm{R}$, et al. Sorafenib in advanced melanoma: a Phase II randomised discontinuation trial analysis. Br J Cancer 2006; 95 (5): 581-6.

26. Kirkwood JM, Bastholt L, Robert C, Sosman J, Larkin J, Hersey P, et al. Phase II, open-label, randomized trial of the MEK1/2 inhibitor selumetinib as monotherapy versus temozolomide in patients with advanced melanoma. Clin Cancer Res 2012; 18 (2): 555-67.

27. Kaufman HL. Melanoma as a model for precision medicine in oncology. Lancet Oncol 2014; 15 (3): 251-3.

28. Chapman PB, Hauschild A, Robert C, Haanen JB, Ascierto $P$, Larkin J, et al. Improved survival with vemurafenib in melanoma with BRAF V600E mutation. N Engl J Med 2011; 364 (26): 2507-16.

29. Sosman JA, Kim KB, Schuchter L, Gonzalez R, Pavlick AC, Weber JS, et al. Survival in BRAF V600-mutant advanced melanoma treated with vemurafenib. N Engl J Med 2012; 366 (8): 707-14.

30. Falchook GS, Long GV, Kurzrock R, Kim KB, Arkenau TH, Brown MP, et al. Dabrafenib in patients with melanoma, untreated brain metastases, and other solid tumours: a phase 1 dose-escalation trial. Lancet 2012; 379 (9829): 1893-901.

31. Hauschild A, Grob JJ, Demidov LV, Jouary T, Gutzmer R, Millward M, et al. Dabrafenib in BRAF-mutated metastatic melanoma: a multicentre, open-label, phase 3 randomised controlled trial. Lancet 2012; 380 (9839): 358-65.

32. Ma XH, Piao SF, Dey S, McAfee Q, Karakousis G, Villanueva J, et al. Targeting ER stress-induced autophagy overcomes BRAF inhibitor resistance in melanoma. J Clin Invest 2014; 124 (3): 1406-17.

33. Montagut C, Sharma SV, Shioda T, McDermott U, Ulman M, Ulkus LE, et al. Elevated CRAF as a potential mechanism of acquired resistance to BRAF inhibition in melanoma. Cancer Res 2008; 68 (12): 4853-61.

34. Van Allen EM, Wagle N, Sucker A, Treacy DJ, Johannessen CM, Goetz EM, et al. The genetic landscape of clinical resistance to RAF inhibition in metastatic melanoma. Cancer Discov 2014; 4 (1): 94-109.

35. Rizos H, Menzies AM, Pupo GM, Carlino MS, Fung C, Hyman J, et al. BRAF inhibitor resistance mechanisms in metastatic melanoma: spectrum and clinical impact. Clin Cancer Res 2014; 20 (7): 1965-77.

36. McArthur GA, Ribas A. Targeting oncogenic drivers and the immune system in melanoma. J Clin Oncol 2013; 31 (4): 499-506.

37. Anforth R, Fernandez-Penas P, Long GV. Cutaneous toxicities of RAF inhibitors. Lancet Oncol 2013; 14 (1): e11-8.

38. Boyd KP, Vincent B, Andea A, Conry RM, Hughey LC. Nonmalignant cutaneous findings associated with 
vemurafenib use in patients with metastatic melanoma. J Am Acad Dermatol 2012; 67 (6): 1375-9.

39. Dalle S, Poulalhon N, Thomas L. Vemurafenib in melanoma with BRAF V600E mutation. N Engl J Med 2011; 365 (15): 1448-9; author reply 50.

40. Zimmer L, Hillen U, Livingstone E, Lacouture ME, Busam K, Carvajal RD, et al. Atypical melanocytic proliferations and new primary melanomas in patients with advanced melanoma undergoing selective BRAF inhibition. J Clin Oncol 2012; 30 (19): 2375-83.

41. Larkin J, Del Vecchio M, Ascierto PA, Krajsova I, Schachter J, Neyns B, et al. Vemurafenib in patients with BRAF(V600) mutated metastatic melanoma: an open-label, multicentre, safety study. Lancet Oncol 2014; 15 (4): 436-44.

42. Sosman J. Molecularly targeted therapy for metastatic melanoma. En: UpToDate. 2014.

43. Flaherty KT, Robert C, Hersey P, Nathan P, Garbe C, Milhem $\mathrm{M}$, et al. Improved survival with MEK inhibition in BRAF-mutated melanoma. N Engl J Med 2012; 367 (2): 107-14.

44. Larkin J, Ascierto PA, Dreno B, Atkinson V, Liszkay G, Maio M, et al. Combined vemurafenib and cobimetinib in BRAF-mutated melanoma. N Engl J Med 2014; 371 (20): 1867-76.

45. Ribas A, Gonzalez R, Pavlick A, Hamid O, Gajewski TF, Daud A, et al. Combination of vemurafenib and cobimetinib in patients with advanced BRAF(V600)-mutated melanoma: a phase 1b study. Lancet Oncol 2014; 15 (9): 954-65.

46. Anforth R, Liu M, Nguyen B, Uribe P, Kefford R, Clements A, et al. Acneiform eruptions: a common cutaneous toxicity of the MEK inhibitor trametinib. Australas J Dermatol 2014; 55 (4): 250-4.

47. Uribe P, Anforth RM, Kefford RF, Fernandez-Penas P. Acneiform eruption in a patient with metastatic melanoma after ceasing combination dabrafenib/trametinib therapy. Melanoma Res 2014; 24 (5): 501-3.

48. Infante JR, Fecher LA, Falchook GS, Nallapareddy S, Gordon MS, Becerra C, et al. Safety, pharmacokinetic, pharmacodynamic, and efficacy data for the oral MEK inhibitor trametinib: a phase 1 dose-escalation trial. Lancet Oncol 2012; 13 (8): 773-81.

49. Long GV, Stroyakovskiy D, Gogas H, Levchenko E, de Braud F, Larkin J, et al. Combined BRAF and MEK inhibition versus BRAF inhibition alone in melanoma. N Engl J Med 2014; 371 (20): 1877-88.

50. Robert C, Karaszewska B, Schachter J, Rutkowski P, Mackiewicz A, Stroiakovski D, et al. Improved overall survival in melanoma with combined dabrafenib and trametinib. N Engl J Med 2015; 372 (1): 30-9.
51. Flaherty KT, Infante JR, Daud A, Gonzalez R, Kefford RF, Sosman J, et al. Combined BRAF and MEK inhibition in melanoma with BRAF V600 mutations. N Engl J Med 2012; 367 (18): 1694-703.

52. Lopez MN, Pereda C, Segal G, Muñoz L, Aguilera R, Gonzalez FE, et al. Prolonged survival of dendritic cell-vaccinated melanoma patients correlates with tumor-specific delayed type IV hypersensitivity response and reduction of tumor growth factor beta-expressing T cells. J Clin Oncol 2009; 27(6): 945-52.

53. Blanchard T, Srivastava PK, Duan F. Vaccines against advanced melanoma. Clin Dermatol 2013; 31(2): 17990.

54. Topalian SL, Hodi FS, Brahmer JR, Gettinger SN, Smith DC, McDermott DF, et al. Safety, activity, and immune correlates of anti-PD-1 antibody in cancer. N Engl J Med 2012; 366 (26): 2443-54.

55. Hodi FS, O'Day SJ, McDermott DF, Weber RW, Sosman JA, Haanen JB, et al. Improved survival with ipilimumab in patients with metastatic melanoma. N Engl J Med 2010; 363 (8): 711-23.

56. Hamid O, Schmidt H, Nissan A, Ridolfi L, Aamdal S, Hansson J, et al. A prospective phase II trial exploring the association between tumor microenvironment biomarkers and clinical activity of ipilimumab in advanced melanoma. J Transl Med 2011; 9: 204.

57. Ascierto PA, Kalos M, Schaer DA, Callahan MK, Wolchok JD. Biomarkers for immunostimulatory monoclonal antibodies in combination strategies for melanoma and other tumor types. Clin Cancer Res 2013; 19 (5): 1009-20.

58. Wolchok JD, Hoos A, O'Day S, Weber JS, Hamid O, Lebbe $\mathrm{C}$, et al. Guidelines for the evaluation of immune therapy activity in solid tumors: immune-related response criteria. Clin Cancer Res 2009; 15 (23): 7412-20.

59. Di Giacomo AM, Biagioli M, Maio M. The emerging toxicity profiles of anti-CTLA-4 antibodies across clinical indications. Semin Oncol 2010; 37 (5): 499-507.

60. Weber JS, Kahler KC, Hauschild A. Management of immune-related adverse events and kinetics of response with ipilimumab. J Clin Oncol 2012; 30 (21): 2691-7.

61. Robert C, Long GV, Brady B, Dutriaux C, Maio M, Mortier L, et al. Nivolumab in previously untreated melanoma without BRAF mutation. N Engl J Med 2015; 372 (4): 320-30.

62. Two-Year Survival and Safety Update in Patients (pts) with Treatment-Naïve Advanced Melanoma (MEL) Receiving Nivolumab (NIVO) or Dacarbazine (DTIC) in CheckMate-066. Presented at the Society for Melanoma Research 2015 International Congress; November 18-21, 2015; San Francisco, CA. 
63. Weber JS, D'Angelo SP, Minor D, Hodi FS, Gutzmer R, Neyns B, et al. Nivolumab versus chemotherapy in patients with advanced melanoma who progressed after anti-CTLA-4 treatment (CheckMate 037): a randomised, controlled, open-label, phase 3 trial. Lancet Oncol 2015; 16 (4): 375-84.

64. Hamid O, Robert C, Daud A, Hodi FS, Hwu WJ, Kefford R, et al. Safety and tumor responses with lambrolizumab (anti-PD-1) in melanoma. N Engl J Med 2013; 369 (2): 134-44.

65. Robert C, Schachter J, Long GV, Arance A, Grob JJ, Mortier L, et al. Pembrolizumab versus Ipilimumab in Advanced Melanoma. N Engl J Med 2015.

66. Ribas AF, Hodi FS, Kefford R, Hamid O, Daud A, Wolchok JD, et al. Efficacy and safety of the anti-PD-1 monoclonal antibody MK-3475 in 411 patients (pts) with melanoma (MEL). ASCO Meeting 2014. J Clin Oncol 32: 5s, 2014 (suppl; abstr LBA9000^).

67. Brahmer JR, Tykodi SS, Chow LQ, Hwu WJ, Topalian SL, Hwu P, et al. Safety and activity of anti-PD-L1 antibody in patients with advanced cancer. N Engl J Med 2012; 366 (26): 2455-65.

68. Freeman-Keller M, Weber JS. Anti-programmed death receptor 1 immunotherapy in melanoma: rationale, evidence and clinical potential. Ther Adv Med Oncol 2015; 7 (1): 12-21.

69. Mahoney KM, Freeman GJ, McDermott DF. The Next
Immune-Checkpoint Inhibitors: PD-1/PD-L1 Blockade in Melanoma. Clin Ther 2015; 37 (4): 764-82.

70. Postow MA, Chesney J, Pavlick AC, Robert C, Grossmann K, McDermott D, et al. Nivolumab and ipilimumab versus ipilimumab in untreated melanoma. $\mathrm{N}$ Engl J Med 2015; 372 (21): 2006-17.

71. Larkin J, Chiarion-Sileni V, Gonzalez R, Grob JJ, Cowey CL, Lao CD, et al. Combined Nivolumab and Ipilimumab or Monotherapy in Untreated Melanoma. N Engl J Med 2015; 373 (1): 23-34.

72. Sznol M, Kluger HM, Callahan MK, Postow MA, Gordon RA, Segal NH, et al. Survival, response duration, and activity by BRAF mutation (MT) status of nivolumab (NIVO, anti-PD-1, BMS-936558, ONO-4538) and ipilimumab (IPI) concurrent therapy in advanced melanoma (MEL). J Clin Oncol 32: 5s, 2014 (suppl; abstr LBA9003^).

73. Wolchok JD, Kluger H, Callahan MK, Postow MA, Rizvi NA, Lesokhin AM, et al. Nivolumab plus ipilimumab in advanced melanoma. N Engl J Med 2013; 369 (2): 122-33.

74. Sosman J. Overview of the management of advanced cutaneous melanoma. En: UpToDate, Atkins MB (Ed), UpToDate, Waltham, MA. (Consultado el 20 de diciembre de 2015). Disponible en: http://www.uptodate.com.

75. Ollila DW, Gleisner AL, Hsueh EC. Rationale for complete metastasectomy in patients with stage IV metastatic melanoma. J Surg Oncol 2011; 104(4): 420-4. 